\title{
Scorpion fluorescence and reaction to light
}

\author{
Douglas D. Gaffin ${ }^{\mathrm{a}, *}$, Lloyd A. Bumm ${ }^{\mathrm{b}}$, Matthew S. Taylor ${ }^{\mathrm{a}}$, Nataliya V. Popokina ${ }^{\mathrm{a}}$, Shivani Mann ${ }^{\mathrm{a}}$ \\ ${ }^{a}$ Department of Zoology, University of Oklahoma, Norman, U.S.A. \\ ${ }^{\mathrm{b}}$ Homer L. Dodge Department of Physics and Astronomy, University of Oklahoma, Norman, U.S.A.
}

\section{A R T I C L E I N F O}

\section{Article history:}

Received 27 July 2011

Initial acceptance 16 August 2011

Final acceptance 20 October 2011

Available online $\mathrm{xxx}$

MS. number: A11-00590

\section{Keywords:}

fluorescence light

orientation

Paruroctonus utahensis

scorpion

sensory

ultraviolet

vision

\begin{abstract}
Scorpions are largely solitary, nocturnal arachnids that glow a bright cyan-green under UV light. The function of this fluorescence is a mystery. Previous studies of four species from three families have shown that scorpion lateral and medial eyes are maximally sensitive to green light (around $500 \mathrm{~nm}$ ) and secondarily to UV (350-400 nm). Scorpions are negatively phototactic, and we used this behaviour to assay the responses of desert grassland scorpions, Paruroctonus utahensis, to $395 \mathrm{~nm}$ UV light, $505 \mathrm{~nm}$ cyan-green light, $565 \mathrm{~nm}$ green light and no light within small, circular arenas. Based on the eye sensitivity data, we predicted maximal response to $505 \mathrm{~nm}$, followed by lower responses to 395 and $565 \mathrm{~nm}$. In our experiments, however, scorpions responded most intensely (abrupt bouts of locomotory activity) to $395 \mathrm{~nm}$ and $505 \mathrm{~nm}$. Next, we ran trials under 395 and $505 \mathrm{~nm}$ on scorpions with their eyes blocked. Scorpions with blocked eyes were much less likely to move under $505 \mathrm{~nm}$ than under $395 \mathrm{~nm}$ and were much less likely to move under $505 \mathrm{~nm}$ than were control animals (those without their eyes blocked). These results suggest an active role for fluorescence in scorpion light detection. Other studies indicate that photosensitive elements in scorpion tails are sensitive to green light. We therefore propose that the cuticle may function as a whole-body photon collector, transducing UV light to cyan-green before relaying this information to the central nervous system. Scorpions may use this information to detect shelter, as blocking any part of the cuticle could diminish the signal.
\end{abstract}

(c) 2011 The Association for the Study of Animal Behaviour. Published by Elsevier Ltd. All rights reserved.
Scorpions are nocturnal arachnids whose cuticle fluoresces a brilliant cyan-green under ultraviolet (UV) light. Scorpion collectors have long used this unusual phenomenon to locate the animals at night (Honetschlager 1965). Two cuticular molecules, beta-carboline and 4-methyl-7-hydroxycoumarin, account for the fluorescence (Stachel et al. 1999; Frost et al. 2001); however, a functional role for fluorescence has not been established.

There are many hypotheses about the role of scorpion fluorescence. One possibility is that the fluorescence serves no behavioural function and that the fluorescing chemicals are simply metabolic by-products (Wankhede 2004). A related idea is that fluorescence is a vestigial trait retained from ancient diurnal species (Frost et al. 2001). Another hypothesis is that the fluorescence serves as a prey lure; sand scorpions sit and wait on the surface or in the entrances of their burrows (Polis 1979, 1980), where insects and other potential prey could be visually drawn towards their fluorescence. A test of this hypothesis, however, suggested that insects actually avoid fluorescing scorpions (Kloock 2005). An untested idea is that fluorescence serves as an aposematic signal. Yet another

\footnotetext{
* Correspondence: D. D. Gaffin, Department of Zoology, University of Oklahoma, Norman, OK 73019, U.S.A.

E-mail address: ddgaffin@ou.edu (D. D. Gaffin).
}

hypothesis is that scorpion fluorescence aids in the recognition of conspecifics, especially during mate-finding behaviour or courtship rituals (Brownell 2001; Kloock 2008). Along these lines, UV-induced fluorescence on female palps in jumping spiders, Cosmophasis umbratica, has been identified as an adaptation in mating rituals (Lim et al. 2007).

Some information exists on scorpion eye structure and function. Scorpions have well-developed eyes; their median eyes appear capable of image formation, and their lateral eyes can identify subtle changes in light magnitude. Both sets of eyes are highly sensitive to light and can putatively detect starlight against the background of the night sky (Schliwa \& Fleissner 1980; Fleissner \& Fleissner 2001). Scorpion median eyes have a primary wavelength sensitivity peak and a secondary plateau (Machan 1968; Fleissner \& Fleissner 2001). The plateau is in the UV range between 350 and $400 \mathrm{~nm}$, where sensitivity is about $65 \%$ of maximum. The ocular sensitivity gradually increases to its maximum around $500 \mathrm{~nm}$. The neurological response steadily drops with wavelengths longer than approximately $520 \mathrm{~nm}$, and the response disappears at wavelengths around $640 \mathrm{~nm}$. Furthermore, scorpions have metasomal elements that are also most sensitive to light in the green range (Zwicky 1968, 1970a, b; Rao \& Rao 1973). Interestingly, 395 nm UV light elicits the strongest fluorescence in scorpions, and desert scorpions fluoresce light with a wavelength of about $500 \mathrm{~nm}$, 
which corresponds to the peak sensitivity of the eyes (Fasel et al. 1997; Kloock 2009).

Fluorescence in scorpions may therefore be related to their vision and increased activity under UV and green light (Camp \& Gaffin 1999; Blass \& Gaffin 2008). Scorpions become active soon after sunset, the only time of night where the sky emits more shorter wavelengths of light (approximately 350-450 $\mathrm{nm}$ ) than longer wavelengths of light (Johnsen et al. 2006). Blass \& Gaffin (2008) showed that scorpions run in spurts and spend less time in light-exposed areas under these shorter ultraviolet wavelengths. Scorpions do not react to longer wavelengths ( $\sim 630 \mathrm{~nm}$ ), but do respond strongly to both $405 \mathrm{~nm}$ and $525 \mathrm{~nm}$. More recently, Kloock et al. (2010) found that under UV light, photo-bleached scorpions with reduced fluorescence made more transitions between exposed and unexposed portions of small arenas compared to fluorescent scorpions.

Inspired by these findings, we further investigated scorpions' behavioural responses to specific wavelengths of light. We tested Paruroctonus utahensis (Williams, 1968) (Scorpiones: Vaejovidae) under 395 nm UV light, 505 nm cyan-green light, 565 nm green light and no light. Based on physiological evidence showing that scorpion eyes are more sensitive to cyan-green light than to UV light (Fleissner \& Fleissner 2001), and that scorpions avoid light (Abushama 1964; Camp \& Gaffin 1999), we predicted P. utahensis would be more active under a $505 \mathrm{~nm}$ wavelength than under a $395 \mathrm{~nm}$ wavelength. Since $505 \mathrm{~nm}$ differs from $565 \mathrm{~nm}$ by only $60 \mathrm{~nm}$, yet differs from $395 \mathrm{~nm}$ by $110 \mathrm{~nm}$, we also expected that responses to $395 \mathrm{~nm}$ and $565 \mathrm{~nm}$ would be roughly equivalent. Our results, however, showed similar activity of scorpions under 395 and $505 \mathrm{~nm}$, and significantly greater activity under these wavelengths compared to $565 \mathrm{~nm}$.

The results of these trials suggest that scorpions are more active under $395 \mathrm{~nm}$ light than would be expected by their ocular sensitivities. As such, we hypothesized that scorpion cuticular fluorescence may be involved in their perception of light. We predicted that scorpions with their eyes blocked should be less active under $505 \mathrm{~nm}$ compared to $395 \mathrm{~nm}$ and compared to animals without their eyes blocked under either wavelength. We found that scorpions with their eyes blocked moved significantly less under $505 \mathrm{~nm}$ compared to the other conditions. Taken together, these results suggest that scorpion fluorescence contributes to scorpion light reception and orientation, and may be adaptive in lightavoidance behaviour, as during the choice to remain in their burrows until light levels decline in the early evening or full moon, or in detecting shelter during predator avoidance.

\section{METHODS}

Animals

We used male and female $P$. utahensis collected during March 2010 and 2011 from a sandy region about $30 \mathrm{~km}$ southeast of Monahans, Texas. In the laboratory, we kept the animals in individual glass jars with about $2-3 \mathrm{~cm}$ of sand covering the bottom. We fed the scorpions one waxworm (Achroia grisella) every 3-4 weeks and watered them twice per week. The room temperature remained within a range of $21-24^{\circ} \mathrm{C}$, and the room lighting followed a 16:8 h light:dark cycle.

\section{Behavioural Apparatus}

We filmed the scorpions from beneath a Plexiglas sheet using an infrared-sensitive camera (Sony Handycam CCD-TRV16 with 'nightshot' feature). The camera was connected to a laptop that ran a video capture program for recording footage (Fig. 1). Scorpions were contained individually in covered $8.75 \mathrm{~cm}$ diameter clear
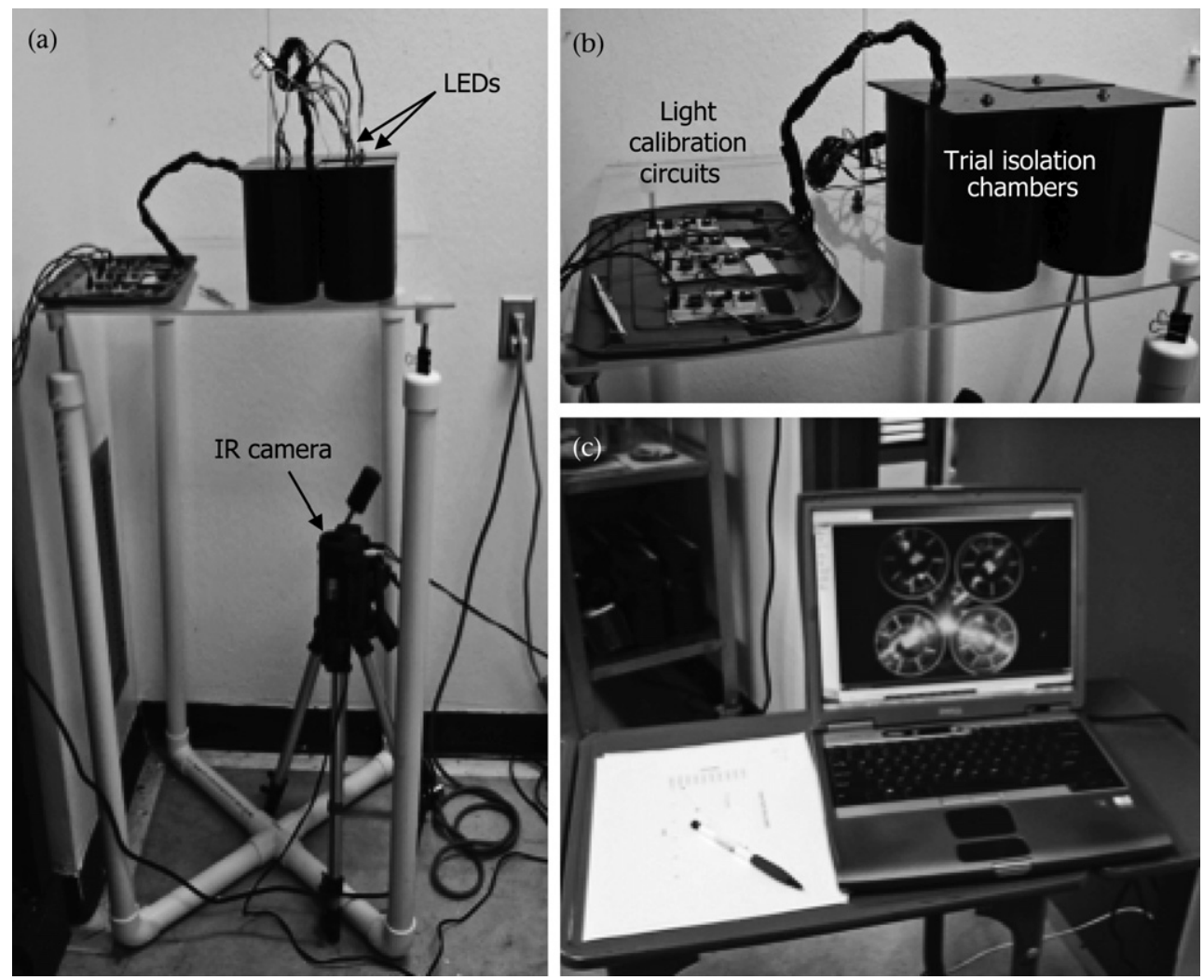

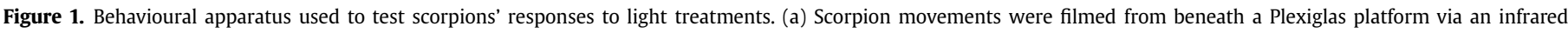

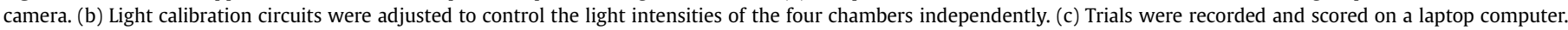


plastic petri dish arenas. To provide a circular running track, we glued a smaller petri dish (5.40 cm diameter) upside down into the centre of the bottom of the larger dish (for details, see Blass \& Gaffin 2008). To reduce reflections the scorpions might see of themselves, we painted the vertical insides of the petri dish track a flat black using Elmer's Painters (Elmer's Products, Inc., Columbus, OH, U.S.A.) paint pens.

We divided the circular track of the petri dish into 16 even segments by thin white lines painted on the bottom using white Elmer's Painters paint pens. A piece of PVC pipe $(10 \mathrm{~cm}$ in diameter and $15 \mathrm{~cm}$ tall) was placed over each petri dish arena. Each pipe was lined on the inside with black construction paper and topped with a $10 \times 10 \times 1 \mathrm{~cm}$ square piece of black Plexiglas that was sealed to the PVC using silicon glue. Each square of the black Plexiglas had a $5 \mathrm{~mm}$ hole drilled into the middle to fit a light-emitting diode (LED). We built op-amp circuits to precisely control and match LED intensities. We used three different $5 \mathrm{~mm}$ LEDs (Super Bright LEDs Inc., Earth City, MO, U.S.A.): ultraviolet (395 nm, $12 \mathrm{~mW} / 15^{\circ}$ ), cyan-green ( $505 \mathrm{~nm}, 9000 \mathrm{mcd} / 18^{\circ}$ ), and yellow-green (565 nm, $\left.1000 \mathrm{mcd} / 20^{\circ}\right)$. We chose these wavelengths because they are key points in the scorpion retinal sensitivity spectrum (Fleissner \& Fleissner 2001). LEDs were connected and swapped using miniature patch cords with hook clips.

\section{Wavelength Response Trials}

We collected all the data for these trials during May 2010 between 2 and $6 \mathrm{~h}$ after the beginning of the dark cycle. We randomly sorted 40 animals into two groups of 20 (10 male, 10 female); these groups were alternated with each trial night, and we let each animal rest at least 2 days between trials. We recorded an equal number of trials each session, and every animal experienced a different lighting condition during each trial. Through the course of the experiment, all scorpions were subjected to each lighting condition (395 nm, $505 \mathrm{~nm}, 565 \mathrm{~nm}$ and no light) and the lights were matched for intensity (see below). We filmed four scorpions at a time, using a randomized schedule to determine the set-up for each taping.

Forty minutes before filming started, the petri dish arenas were cleaned with a tissue and $70 \%$ ethanol. Twenty minutes later, we moved the animals from their jars to the arenas. Fifteen minutes before filming, we moved the scorpions into the filming room, placed them on the Plexiglas stage, covered them with the black PVC pipes, and inserted the appropriate inactivated LEDs for each animal. The scorpions were left undisturbed for $15 \mathrm{~min}$ to acclimate to the dishes and the darkness. We then turned on and focused the infrared (IR) video camera, turned on the LEDs, and started the video capture program to begin recording. We filmed the scorpions for $10 \mathrm{~min}$. After recording, we placed the scorpions back in their jars.

\section{Eyes-blocked Trials}

We collected all the data for these trials during May 2011 between 2 and $5 \mathrm{~h}$ after the beginning of the dark cycle. There were six treatment groups in this set of trials. We used a total of 40 animals (both males and females); half of the animals experienced $395 \mathrm{~nm}$ light, and the other half experienced $505 \mathrm{~nm}$ light. The lights were matched for intensity to $6.5 \mu \mathrm{W} / \mathrm{cm}^{2}$ (see below). Each group of 20 animals experienced three test conditions in randomized order: (1) no manipulation (control treatment); (2) sticky tape on the dorsal prosoma (sham treatment); (3) sticky tape plus a small piece of aluminium foil $\left(\sim 1 \mathrm{~cm}^{2}\right)$ to cover the medial and lateral eyes (eyes-blocked treatment; see Results, inset of Fig. 4b). The sticky tape consisted of adhesive sticky tabs (Ted Pella, Inc.,
Redding, CA, U.S.A.), rolled into $3 \mathrm{~mm}$ balls, and arranged in a triangle on the prosoma around the medial eyes (one in front and two behind). We used a spectrophotometer (described below) to check the transmittance of green and UV light and found that the sticky tape did not block green light, but did block some of the UV; it showed no fluorescence under UV. The aluminium foil shields and/or sticky tape were placed on the animals approximately $1 \mathrm{~h}$ before the beginning of the trials. We ran four trials at a time, and the trials lasted for $10 \mathrm{~min}$; the protocol for the trials was the same as for the wavelength response trials. Scorpions were given $48 \mathrm{~h}$ to rest between treatments; we filmed five trials per night for six consecutive nights to generate 120 total trials.

\section{Light Calibrations}

It was crucial to calibrate the LEDs to the same relative intensity so that the scorpions were exposed to the same irradiance, regardless of the wavelength. In the wavelength response trials, we used a Melles Griot Broadband Power/Energy Meter (13PEM001) to measure the power ( $\mathrm{mW}$ ) of each LED on the same circuit; we then converted the measurements to irradiance $\left(\mu \mathrm{W} / \mathrm{cm}^{2}\right)$. We measured power per distance as well as power per voltage to ensure the linearity of the device's measurements and of the lights' responses to the voltage adjustments. Based on results of a pilot study, we selected an irradiance value of $6.5 \mu \mathrm{W} / \mathrm{cm}^{2}$ for the experiments, which is approximately equivalent to irradiance at late dusk or three times the intensity of full moon light $(2.1 \mu \mathrm{W} /$ $\mathrm{cm}^{2}$; Johnsen et al. 2006). We calculated the voltages required to obtain this intensity for each LED. We adjusted the voltages to account for the slight variations among our four circuits. We used a Pasco OS-9152B photometer (which is accurate for comparing light intensities of the same wavelength) to further adjust voltages and match the wavelength intensities among circuits. For the eyesblocked trials, we used an Ocean Optics spectrophotometer with optical fibre and cosine corrector (USB2000 + UV - VIS) to adjust the LED intensities to $6.5 \mu \mathrm{W} / \mathrm{cm}^{2}$.

\section{Analysis}

\section{Wavelength response trials}

We recorded the first 20 crossings for each animal by pausing the video at each crossing and recording the video timestamp in seconds. We then calculated the intercrossing intervals (the time between each crossing; ICI), excluding ICIs that were 45 s or longer. We considered a trial valid if the scorpion had at least 12 ICIs shorter than $45 \mathrm{~s}$. We chose these parameters based on Blass \& Gaffin (2008), where the petri dish arenas were divided into eight equal segments and trials were considered valid with at least five ICIs shorter than $90 \mathrm{~s}$. Since we increased the resolution of the scoring by increasing the number of segments to 16 , we felt it appropriate and conservative to increase the number of required ICIs to 12 and reduce the required crossing time to $45 \mathrm{~s}$. Together, we felt this allowed the animal adequate time and space to register an appropriate behavioural response. We analysed the data by looking at time until first crossing, mean and median ICI length, and relative frequencies of each ICI length. We found that under the irradiance levels of these experiments, and similar to the studies of Blass \& Gaffin (2008), that UV- and green-stimulated animals tended to run in brief spurts. As such, simply counting the number of line crossings during the $10 \mathrm{~min}$ trials was not an effective measure of response. Animals under no light and $565 \mathrm{~nm}$ conditions tended to walk slower and steadier than the UV- and green-stimulated animals and could therefore cross a similar number of lines during the $10 \mathrm{~min}$ trials as the stimulated animals that ran in quick bursts with longer pauses. We found that transforming the data to 
indicate the frequencies of interline crossings of various durations was the most sensitive measure of response. In particular, we analysed the frequency of ICIs of duration of 1,2 and $3 \mathrm{~s}$ per trial. We conducted a Friedman nonparametric repeated measures ANOVA test using InStat 3 statistical software (Graph Pad Software, Inc., San Diego, CA, U.S.A.) to test for overall differences among the four sample groups: $395 \mathrm{~nm}, 505 \mathrm{~nm}, 565 \mathrm{~nm}$ and no light. Tests for normality failed for all measures except the distribution of trial ICI means. We used a Dunn's multiple comparisons test for post hoc analysis of those measures with an overall significance value of $P<0.05$. Our null hypothesis was that scorpions' responses would not differ significantly between light conditions.

\section{Eyes-blocked trials}

The locomotory behaviour of eyes-blocked animals under $505 \mathrm{~nm}$ was noticeably suppressed, which meant that it was not possible to apply the same 12-line crossing threshold for these trials. Instead, we scored all line crossings for all animals during their 10 min trials and calculated median ICI for each. Animals that did not cross a line received a score of $600 \mathrm{~s}$ (the duration of the trial). Because tests for normality failed for all treatment groups, we again used the Friedman nonparametric repeated measures ANOVA to test for overall significant differences between treatments within each light regime. We used a Dunn's multiple comparisons test for post hoc analysis if the overall treatment significance was $P<0.05$. Our null hypothesis was that there would be no significant difference in response between the treatments. We conducted a followup analysis based on the criterion of movement (one or more lines crossed) versus no movement (no lines crossed). We used Fisher's exact test to compare movement in eyes-blocked animals under 395 and $505 \mathrm{~nm}$.

\section{RESULTS}

\section{Wavelength Response Trials}

Most trials (70\%) met the criteria for validity (12 or more ICIs of less than $45 \mathrm{~s}$ ). Animal movements were noticeably sporadic under certain wavelengths of light compared to no-light trials. The distribution of the first 20 crossings for the $10 \mathrm{~min}$ trials for scorpions under $395 \mathrm{~nm}$ and no light showed that stimulated animals (e.g. $395 \mathrm{~nm}$ ) had a disproportionate number of crossings early in their excursions compared to no-light controls (Fig. 2).

Twenty of the 40 animals had valid trials for all four light conditions ( $395 \mathrm{~nm}, 505 \mathrm{~nm}, 565 \mathrm{~nm}$, no light) and formed the basis of the repeated measures statistical analyses for the wavelength response trials. Mean time to first crossing did not differ significantly between the wavelengths tested (Table 1), and there was no difference in male and female behaviour in these trials. However, we found a significant difference between the averages of the trial ICI means and medians (Table 1). In both cases, Dunn's multiple comparisons test showed significant differences between $395 \mathrm{~nm}$ and $565 \mathrm{~nm}(P<0.001)$ and between $505 \mathrm{~nm}$ and $565 \mathrm{~nm}$ $(P<0.01)$.

While the mean and median data indicated that the $395 \mathrm{~nm}$ and $505 \mathrm{~nm}$ treatments were significantly different from the $565 \mathrm{~nm}$ treatment, they did not differ significantly from the no-light controls. This is because the sporadic behaviour of lightstimulated animals skewed their ICI distributions towards ICIs of shorter duration. Comparison of the mean percentage frequency of ICIs of duration 1-10 s for each light condition revealed a significant difference between light conditions for ICI 1 (Friedman test: $P=0.0004$; Fig. 3). Dunn's multiple comparisons test showed

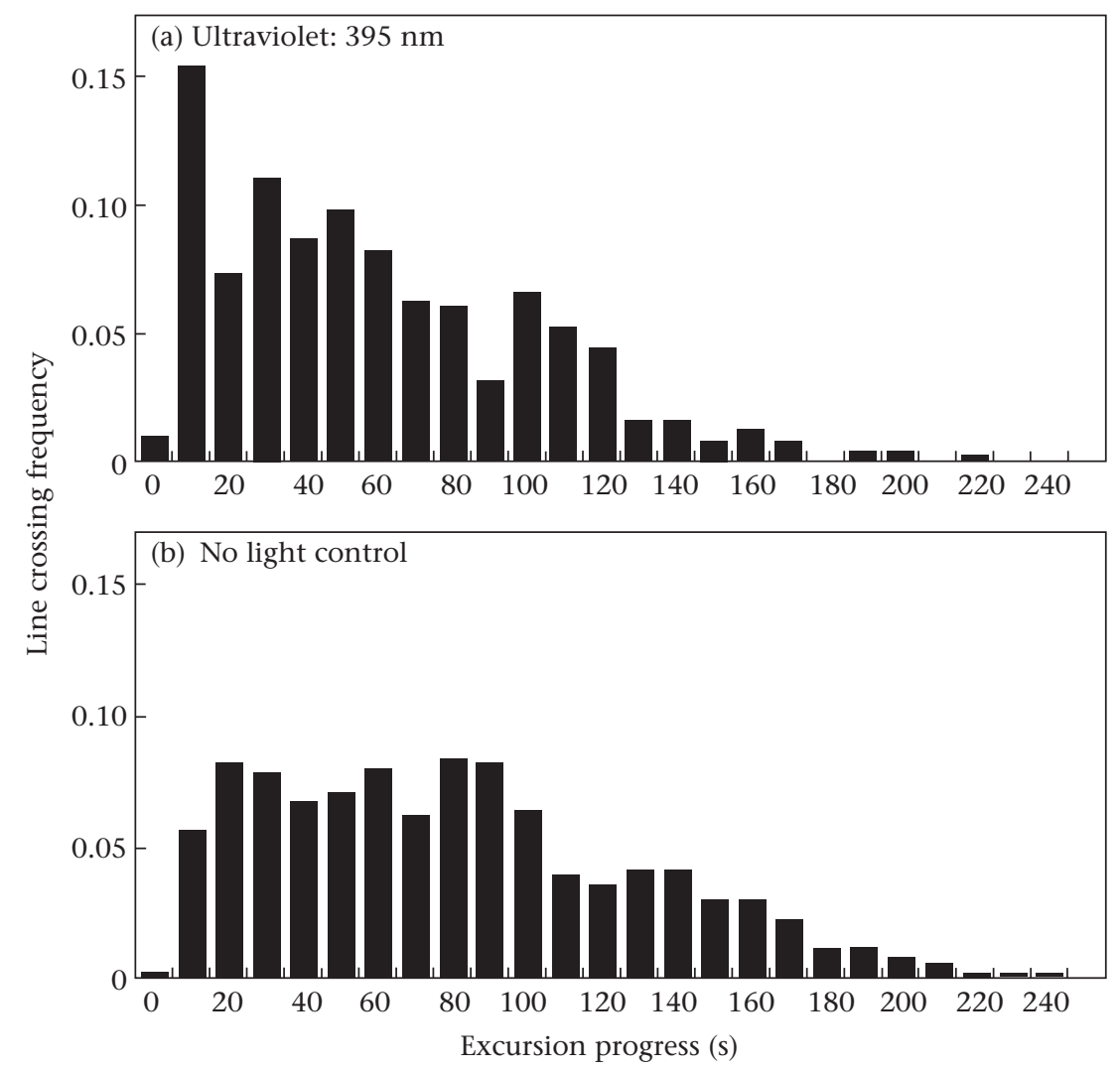

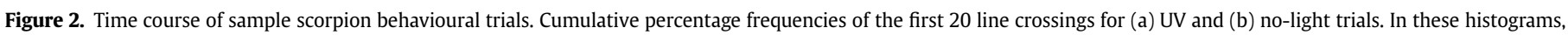
the first line crossing has been set to time zero. 
Table 1

Summary of scorpions' responses during light trials

\begin{tabular}{llll}
\hline $\begin{array}{l}\text { Light } \\
\text { treatment }\end{array}$ & $\begin{array}{l}\text { Mean } \pm \text { SE time } \\
\text { to first crossing }(\mathrm{s})\end{array}$ & $\begin{array}{l}\text { Mean } \pm \text { SE } \\
\text { mean ICI }(\mathrm{s})\end{array}$ & $\begin{array}{l}\text { Mean } \pm \text { SE } \\
\text { median ICI (s) }\end{array}$ \\
\hline $395 \mathrm{~nm}$ & $38.6 \pm 11.4$ & $4.50 \pm 0.75^{\mathrm{b}}$ & $5.86 \pm 0.73^{\mathrm{b}}$ \\
$505 \mathrm{~nm}$ & $49.5 \pm 13.6$ & $4.85 \pm 0.80^{\mathrm{a}}$ & $6.54 \pm 0.79^{\mathrm{a}}$ \\
$565 \mathrm{~nm}$ & $53.6 \pm 15.2$ & $8.25 \pm 0.74^{\mathrm{a}, \mathrm{b}}$ & $9.62 \pm 0.77^{\mathrm{a}, \mathrm{b}}$ \\
No light & $53.4 \pm 26.7$ & $6.28 \pm 0.49$ & $7.83 \pm 0.48$ \\
Friedman $\chi^{2}$ & 7.721 & 18.497 & 20.813 \\
$N$ & 20 & 20 & 20 \\
$P$ & 0.0521 & 0.0003 & 0.0001 \\
\hline
\end{tabular}

ICI: intercrossing interval. Within each column, different superscript letters denote significant differences between treatments $\left(\mathrm{a}={ }^{*} \mathrm{P}<0.01 ; \mathrm{b}={ }^{* *} \mathrm{P}<0.001\right)$.

significant differences between $395 \mathrm{~nm}$ and no light $(P<0.01)$, $395 \mathrm{~nm}$ and $565 \mathrm{~nm}(P<0.05)$, and $505 \mathrm{~nm}$ and no light $(P<0.05)$ for ICI 1 . There was no difference between treatments for ICI 2 , and there was a marginally significant difference between treatments for ICI $3(P=0.0472)$; however, none of the pairwise treatment comparisons for ICI 3 were significantly different.

\section{Eyes-blocked Trials}

The mean percentage ICI frequencies of control and shamtreated animals under 395 and $505 \mathrm{~nm}$ (Fig. 4) were consistent with those in the 395 and $505 \mathrm{~nm}$ wavelength response trials (Fig. 3), suggesting that these animals were behaving similarly to the animals in the previous set of experiments.

Blocking of scorpion eyes significantly altered their behaviour under cyan-green light, but not as strongly as under UV light
(Fig. 4b). For both the 395 and $505 \mathrm{~nm}$ trials, the variation among treatment medians was statistically significant (Friedman test: $395 \mathrm{~nm}$ trials: $\chi_{3}^{2}=8.000, N=16, P=0.0183 ; 505 \mathrm{~nm}$ trials: $\left.\chi_{3}^{2}=10.423, N=15, P=0.0055\right)$. Dunn's multiple comparisons test also showed significant differences between control, sham and eyes-blocked treatments within each light regime. In both sets of trials, Dunn's test adjusted the significance cutoff for pairwise comparisons from $P=0.05$ to $P=0.0062$. For the 395 trials, the sham and eyes-blocked treatments differed significantly from each other $(P=0.0047)$, but neither treatment differed significantly from the control treatment $(P=0.0086$ and $P=0.84$, respectively). For the $505 \mathrm{~nm}$ trials, the control and sham treatments differed significantly from the eyes-blocked treatment $(P=0.0025$ and $P=0.0035$, respectively), but did not differ significantly from each other $(P=0.917)$. Animals with eyes blocked moved significantly less under $505 \mathrm{~nm}$ than under $395 \mathrm{~nm}$ (Friedman test: $\chi_{3}^{2}=5.4270$, $P=0.032$; Fig. 4c).

\section{DISCUSSION}

Scorpions moved in sporadic bursts under cyan-green and UV wavelengths of light compared to yellow light and no light. Furthermore, their response to UV was greater than what would be expected based on previously published physiological studies of retinal sensitivity. Also, scorpions significantly altered their behaviour under green light when their eyes were blocked by foil compared to scorpions without their eyes blocked. The behaviour of eyes-blocked scorpions under UV was less affected.

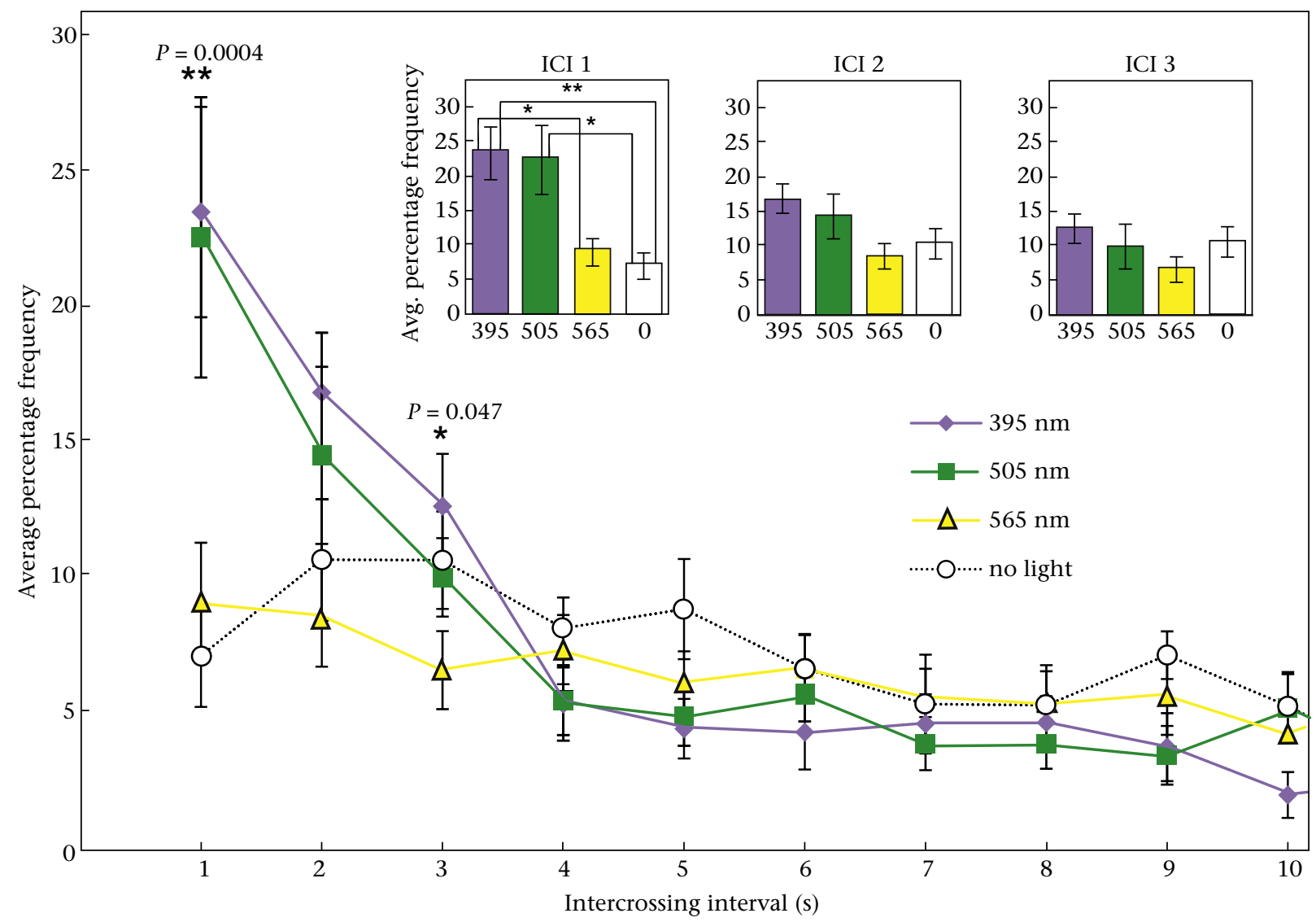

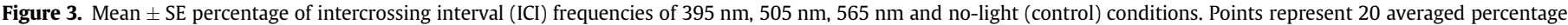

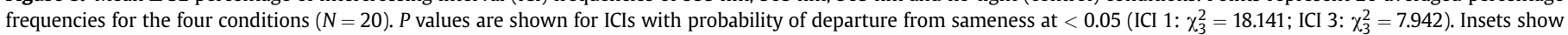
statistical comparisons between wavelengths for the first three ICIs $\left({ }^{*} P<0.05\right.$; $\left.{ }^{* *} P<0.01\right)$. 

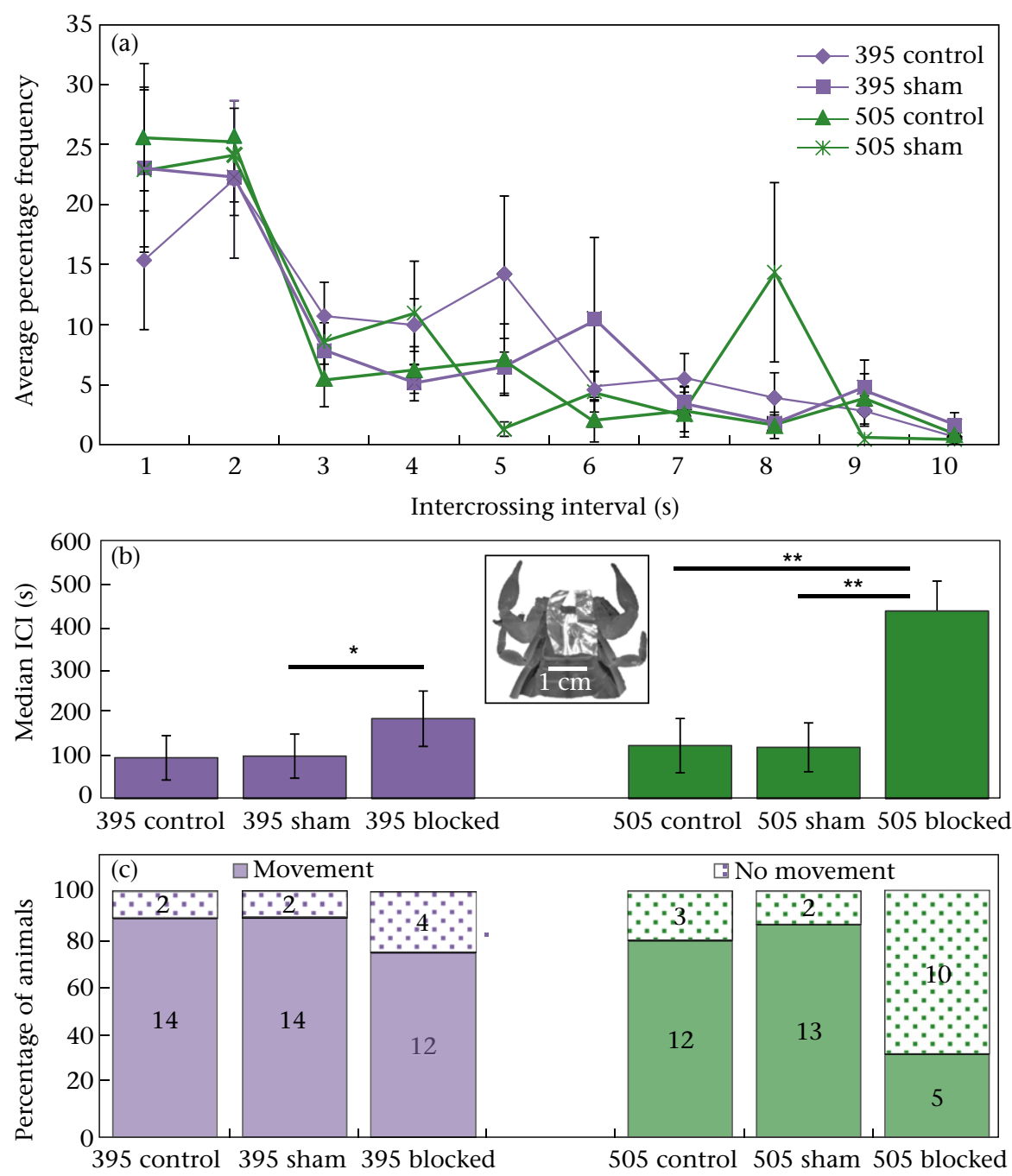

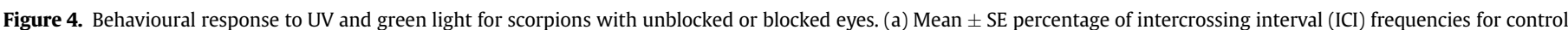

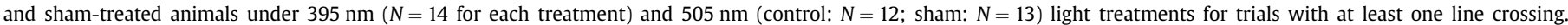

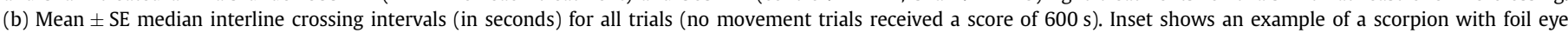

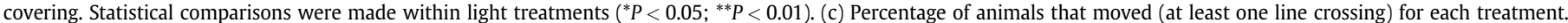
Numbers inside bars indicate the number of animals that did and did not move in each treatment.

\section{Scorpion Fluorescence: a Whole-body UV Photon Collector?}

Since scorpion cuticles fluoresce green under UV light, and the median eyes respond maximally to green and secondarily to UV (Fleissner \& Fleissner 2001), we hypothesize that scorpions' fluorescence may be related to their perception of light. Furthermore, because their tails are maximally sensitive to green wavelengths of light (Rao \& Rao 1973), it would be interesting to learn whether ventral nerve cord ganglia are responsive to green light or green cuticular fluorescence induced by UV. If so, this could set up a system where the cuticle is acting as a whole-body UV photon collector, which transduces shorter UV wavelengths to longer green wavelengths $(\sim 500 \mathrm{~nm})$ before relaying to the central nervous system. This could functionally expand the receptive surface area for UV transduction by many orders of magnitude.

Still, there are challenges with this idea because the simplest way to gain this information would be from UV light directly. However, the scorpion metasoma does not appear to have UV receptors (Rao \& Rao 1973), and scorpions appear to have only one type of photoreceptor pigment in their eyes (Fleissner \& Fleissner 2001). Yet, scorpion lateral eyes show a major peak of excitation in the UV range and a smaller peak in the green range, whereas their medial eyes have a main peak in the green, with a smaller plateau (about $50 \%$ of the green response magnitude) in the UV (Machan 1968; Fleissner \& Fleissner 2001). Kloock et al. (2010) suggested that the elevated responses to UV may actually be a response to green light induced by fluorescence. This, of course, raises the question of whether there is adequate UV in the night sky for this idea to work. The amount of UV available at dusk is about 100 times the amount available in moonless, starlight conditions (Johnsen et al. 2006). Furthermore, only 2\% of UV light is emitted as green fluorescence (Fleissner \& Fleissner 2001). Nevertheless, nearly the entire body of a scorpion fluoresces, and this cuticular surface area would increase the area of UV interception at least a thousand-fold relative to the surface area of the eyes. If a dispersed set of neural elements relayed this subtle stimulation centrally, then a sensitive comparator of left/right illumination seems possible.

Such a system may be adaptive in helping these nocturnal animals find shelter. Sand scorpions in their open sand habitats are particularly vulnerable to predators such as owls and rodents. In the field, we often find $P$. utahensis under isolated twigs or blades of 
grass amid expanses of open sand (Fig. 5). If UV light (from starlight, moonlight, or refracted sunlight) is transduced by the cuticle to green and relayed uniformly to the central nervous system, then blocking any part of the cuticle would diminish the signal to that part of the nervous system and cause the animal to turn towards the shaded portion and move beneath the shelter.

A prediction in line with the whole-body UV photon collector hypothesis is that animals with their eyes blocked should show a diminished behavioural response under green light compared to normal animals, but be less affected under UV. We did find slowed activity of eyes-blocked animals under UV light, which did not support our hypothesis. However, animals with blocked eyes under green light were strikingly less active compared to normal and sham-treated animals under green light. Also, in support of our hypothesis, when we analysed the responses of eyes-blocked animals (395 $\mathrm{nm}$ and $505 \mathrm{~nm}$ treatments) based on movement, or lack thereof, animal activity significantly deviated from expected only under green light; only 5 of the 15 eyes-blocked animals moved under green light.

It is difficult to interpret these results. It is possible that animals with their eyes blocked under green light were comparing photo intensity between their prosomal and metasomal photoreceptors and were induced to remain motionless under the perceived shelter covering their heads. Another possibility relates to the fact that the green fluorescence is of much lower energy than the direct green light delivered by the LED. When the LED intensities are matched as they were in our trials, the magnitude of the differential between the eyes and the rest of the body would therefore be much greater under green light than under UV. With this greatly reduced differential, the UV-stimulated animal may not perceive 'shelter' at all. A useful follow-up study would be to increase the UV or decrease the green so that the central nervous system (CNS) receives the same number of photons under both conditions.

The behaviour of eyes-blocked UV animals is even more difficult to interpret. The somewhat reduced activity of these animals may have been a product of the aluminium foil blocking a significant portion of the prosomal cuticle, thereby reducing the putative fluorescence input to the CNS. Alternatively, it is possible that green light striking the eyes is more important than UV when it comes to light avoidance; perhaps the high response to UV light in the wavelength response trials was partly due to scorpions seeing their

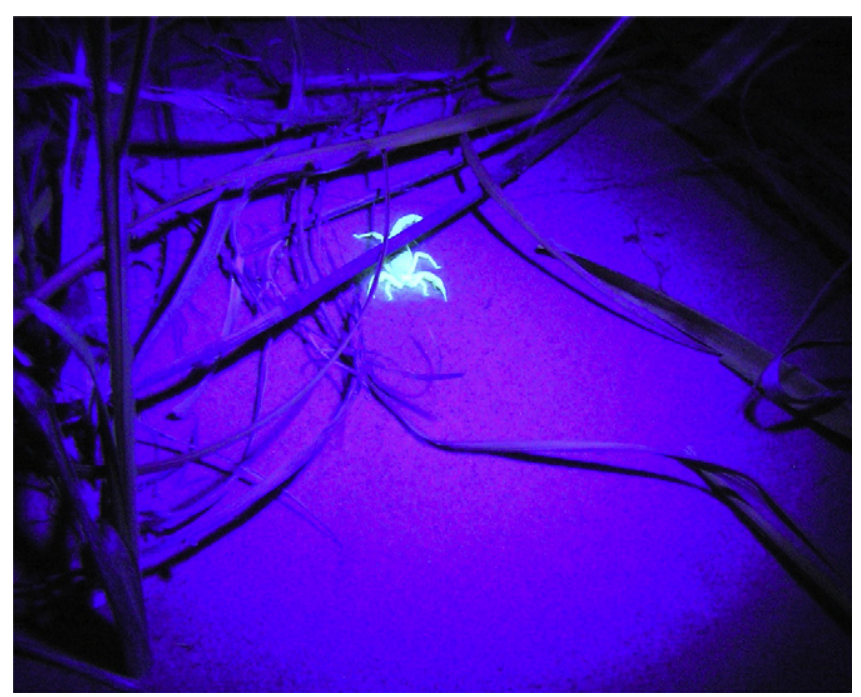

Figure 5. Photo of a female $P$. utahensis at night under a small stem in the field. The female was photographed under UV light at the collection site about $30 \mathrm{~km}$ southeast of Monahans, Texas, U.S.A. Photo: M. Hoefnagels. own green fluorescence. Along these lines, the reduced $395 \mathrm{~nm}$ response could have been caused by leakage of some of the green fluorescence under the foil that reflected to the eyes. Another possibility is that scorpions detect shading as a change in light stimulation across various parts of the body. If the low-energy, green fluorescence is detected generally by the body and relayed to the CNS, and the foil was stationary on the animal's prosoma, then there was no differential in body stimulation and the eyesblocked animals therefore moved similarly to control animals. To investigate this possibility, an arena with a small, stick-sized area blocking the overhead light would be useful. In this assay, we would expect animals under UV to slow their locomotion as they encountered the shade.

Additional studies are needed to test the whole-body UV photon collector hypothesis. For example, we would predict that scorpions' UV sensitivity would be reduced or extinguished if their fluorescence were compromised. Kloock (2009) developed a method for photo-bleaching scorpions through prolonged UV exposure. Animals treated in this way made more transitions between light and dark areas of a behavioural arena under UV light compared to unbleached control animals (Kloock et al. 2010). An alternative method would be to reduce the fluorescence by applying a UV-blocking agent such as sunscreen. We have tried both the photo-bleaching and sunscreen approaches and found that fluorescence is diminished in both cases. We also found that sunscreen with a high SPF value reduces UV transmission while allowing green to penetrate. However, both photo-bleaching and sunscreen compromised scorpion health. We are experimenting with applying the sunscreen to clear tape that can then be applied to the animal's body. An alternative test could involve fashioning a piece of UV-blocking plastic to cover most of the animals' bodies compared to animals with plastic that allows UV transmission. Again, we would predict diminished response for animals under UV and no diminished response for animals under green. Still, this manipulation does not separate a direct response to UV from a response mediated by fluorescence, since the UV is prevented from accessing the cuticle to begin with. Photo-bleaching, or other means of altering molecules responsible for the fluorescence, may help distinguish between these two possibilities.

So far, the clues are converging in favour of a functional role for scorpion fluorescence related to their perception of light. However, many additional studies are warranted, including tests under light of different intensity levels. Our experiments simulated late dusk conditions. What happens when the intensity is lowered systematically to pure starlight conditions? Do the behaviours reported here still persist? What is the intensity at which the behavioural differences vanish? Other behavioural tests could also be useful. For example, focusing UV or green light on various scorpion body parts might yield important behavioural clues to the validity of the whole-body UV photon collector hypothesis. Finally, it would be useful to record electrophysiologically from ventral nerve ganglia of normal animals to see whether a sensitivity to UV light and green light is present. Repeating such tests on photo-bleached animals should show a diminished response to UV, but no change in the green light response.

\section{Acknowledgments}

We thank Dr Douglas Mock, Dr Ingo Schlupp, Dr Mariëlle Hoefnagels, Dr Eric Abraham, Elise Knowlton, Andrea Jordan and Tristan Barker for valuable advice, technical support and consultation services. Thanks also to Jay Vinnedge for assistance with the eye-block behavioural trials. Special thanks go to Greg Blass for making the initial forays into scorpion UV-stimulated behaviour. We also specially thank the referees for many thoughtful 
suggestions on improving the quality of the manuscript. This work was supported in part by funds from the Undergraduate Research Opportunity Program at the University of Oklahoma and the Life account (OU Foundation). These experiments complied with current laws governing animal care and use in the United States.

\section{References}

Abushama, F. T. 1964. On the behavior and sensory physiology of the scorpion Leiurus quinquestriatus. Animal Behaviour, 12, 140-153.

Blass, G. R. C. \& Gaffin, D. D. 2008. Light wavelength biases of scorpions. Animal Behaviour, 76, 365-373.

Brownell, P. H. 2001. Sensory ecology and orientational behaviors. In: Scorpion Biology and Research (Ed. by P. H. Brownell \& G. A. Polis), pp. 159-183. Oxford: Oxford University Press.

Camp, E. A. \& Gaffin, D. D. 1999. Escape Behavior mediated by negative phototaxis in the scorpion Paruroctonus utahensis (Scorpiones, Vaejovidae). Journal of Arachnology, 27, 679-684.

Fasel, A., Muller, P.-A., Suppan, P. \& Vauthey, E. 1997. Photoluminescence of the African scorpion Pandinus imperator. Journal of Photochemistry and Photobiology B: Biology, 39, 96-98.

Fleissner, G. \& Fleissner, G. 2001. Night vision in desert scorpions. In: Scorpions 2001; In Memoriam Gary A Polis (Ed. by V. Fet \& P. A. Selden), pp. 317-324. Burnham Beeches, Bucks: British Arachnological Society.

Frost, L. M., Butler, D. R., O'Dell, B. \& Fet, V. 2001. A coumarin as a fluorescent compound in scorpion cuticle. In: Scorpions 2001; In Memoriam Gary A Polis (Ed. by V. Fet \& P. A. Selden), pp. 363-368. Burnham Beeches, Bucks: British Arachnological Society.

Honetschlager, L. D. 1965. A new method for hunting scorpions. Turtox News, 43, 69.

Johnsen, S., Kelber, A., Warrant, E., Sweeney, A. M., Widder, E. A., Lee, R. L., Jr. \& Hernández-Andrés, J. 2006. Crepuscular and nocturnal illumination and its effects on color perception by the nocturnal hawkmoth Deilephila elpenor Journal of Experimental Biology, 209, 789-800.

Kloock, C. T. 2005. Aerial insects avoid fluorescing scorpions. Euscorpius, 21, 1-7.

Kloock, C. T. 2008. A comparison of fluorescence in two sympatric scorpion species. Journal of Photochemistry and Photobiology B: Biology, 91, 132-136.

Kloock, C. T. 2009. Reducing scorpion fluorescence via prolonged exposure to ultraviolet light. Journal of Arachnology, 37, 368-370.

Kloock, C. T., Kubli, A. \& Reynolds, R. 2010. Ultraviolet light detection: a function of scorpion fluorescence. Journal of Arachnology, 38, 441-445.

Lim, M. L. M., Land, M. F. \& Li, D. 2007. Sex-specific UV and fluorescence signals in jumping spiders. Science, 315, 481.

Machan, L. 1968. Spectral sensitivity of scorpion eyes and the possible role of shielding pigment effect. Journal of Experimental Biology, 49, 95-105.

Polis, G. A. 1979. Prey and feeding phenology of the desert sand scorpion Paruroctonus mesaensis (Scorpionida: Vaejovidae). Journal of Zoology, 188 333-346.

Polis, G. A. 1980. Seasonal patterns and age-specific variation in the surface activity of a population of desert scorpions in relation to environmental factors. Journal of Animal Ecology, 49, 1-18.

Rao, G. \& Rao, K. P. 1973. A metasomatic neuronal photoreceptor in the scorpion. Journal of Experimental Biology, 58, 189-196.

Schliwa, M. \& Fleissner, G. 1980. The lateral eyes of the scorpion, Androctonus australis. Cell and Tissue Research, 206, 95-114.

Stachel, S. J., Stockwell, S. A. \& Van Vranken, D. L. 1999. The fluorescence of scorpions and cataractogenesis. Chemical Biology, 6, 531-539.

Wankhede, R. A. 2004. Extraction, isolation, identification and distribution of soluble fluorescent compounds from the cuticle of scorpion (Hadrurus arizonensis). M.S. thesis, Marshall University, Huntington, West Virginia.

Zwicky, K. T. 1968. A light response in the tail of Urodacus, a scorpion. Life Sciences 7, 257-262

Zwicky, K. T. 1970a. The spectral sensitivity of the tail of Urodacus, a scorpion. Experientia, 26, 317.

Zwicky, K. T. 1970b. Behavioral aspects of the extraocular light sense of Urodacus, a scorpion. Experientia, 26, 747-748. 